\title{
INSTABILITIES IN PRESSURE CONFINED BEAMS AND MORPHOLOGY OF EXTENDED RADIO SOURCES
}

\author{
A.Ferrari, S.Massaglia, E.Trussoni and L.Zaninetti \\ Istituto di Cosmo-geofisica del C.N.R., Torino, Italy \\ Istituto di Fisica Generale dell'Universita', Torino, Italy
}

Several authors have suggested that radio jet morphologies resolved in extragalactic sources are the effects of large-scale Kelvin-Helmholtz instabilities in high-speed, pressureconfined fluid beams ejected from parent active galactic nuclei (Ferrari et al. 1978,1979,1981; Hardee 1979;Benford et al. 1980). In particular results from studies for cylindrical geometries indicate how to connect the "wiggles" (observed in 3C449, NGC 6251, M87 and Cen A) with helical perturbations and the "knots" (observed in NGC 315, M87, Cen Aetc.) with pinching modes. Correspondingly small scale MHD perturbations, generated by the same instability or nonlinear cascade processes, are efficient in accelerating relativistic electrons via stochastic scatterings (Lacombe 1977; Ferrari et al. 1979). This picture may satisfy both the requirements for in situ re-acceleration and the intrinsic correlation between morphology and emission .

While most of the theoretical results so far obtained have been worked out in the linear regime, application to observed morphologies requires a discussion of the level at which the instability saturates before disrupting the beams. For this we may consider the main dissipation mechanisms in coll isionless plasmas: a) interactions of unstable modes with both thermal and suprathermal components of the plasma distribution function; b) nonlinear mode-mode interactions; c) formation of shocks. In the following we assume, consistently with instability calculations, that the fastest growing modes have wavenumber $\mathrm{k} \sim 1$ /a where a is the beam radius, and their typical frquency is $\omega \sim \mathrm{kv}_{\text {beam }}$. In the linear regime (small amplitude perturbations) instability saturates if

$$
\dot{\mathrm{E}}_{\mathbf{a b s}}>\dot{\mathrm{E}}_{\mathbf{K} \mathbf{H}} \sim \frac{\delta \mathrm{B}^{2} / 4 \pi}{\tau_{\mathbf{K} \mathbf{H}}}
$$

where $\dot{\mathrm{E}}_{\mathbf{a b s}}=$ energy absorption rate of interaction of MHD modes with plasma particles, $\mathbf{E}_{\mathbf{K} \mathbf{H}}=$ instability energy release rate and $\tau_{\mathbf{K} \mathbf{H}}=\left(\mathrm{kv}_{\mathbf{S}} a\right)^{-1}=$ linear time scale of instability; numerical calculations show $a \cong 0.1 \div 1$. For supersonic beams the most effective absorption process is direct Fermi- acceleration of suprathermal particles (namely synchrotron emitting electrons); in fact for flows with Mach numbers

$$
\mathbf{M} \gtrsim 4 \cdot 10^{2} \frac{a}{v_{\mathbf{o} 8}} \quad, \quad \mathbf{M}=\mathrm{v}_{\text {beam }} / \mathrm{v}_{\mathbf{s}}
$$

$\left(\mathrm{v}_{\mathbf{0 8}}=\mathrm{v}_{\text {beam }} /\left(10^{8} \mathrm{~cm} / \mathrm{s}\right)\right)$ Eq (1) can be satisfied for $\delta \mathrm{B}^{2} \ll \mathrm{B}^{2}$. For lower Mach numbers saturation may be provided (still in linear regime) by mode-mode interactions; both mode coalescence $\left(\omega_{1}+\omega_{2} \rightarrow\left\{\hat{x}_{3}\right)\right.$ and decay $\left(\omega_{1} \rightarrow \omega_{2}+\omega_{3}\right)$ drag energy away from the fastest growing mode $(\mathrm{ka} \sim 1)$. However decay would create long wavelength modes incompatible with geometrical conditions; at the same time for not-too-high magnetic fields 
$\left(v_{A} \lesssim v_{s}\right)$ coalescence is generally more efficient. This transfers the instability energy to short wavelengths via a cascade process and long wavelength modes cannot grow above $\delta \mathrm{B}^{2}<\mathrm{B}^{2}$. Namely Eq. (1) can be fulfilled as long as

$$
\mathrm{v}_{\mathrm{A}} \gtrsim 2 \mathrm{v}_{\mathrm{s}} a
$$

Short wavelengths may then easily dissipate their energy either via synchrotron electron acceleration or via nonlinear Landau damping on thermal particles.

Finally for transonic flows, $\mathrm{M} \gtrsim 1$, and for $\mathrm{v}_{\mathbf{A}} \lesssim \mathrm{v}_{\mathbf{s}}$, MHD modes cannot be stabilized in the linear regime. However the perturbation amplitude cannot exceed $\sim \lambda / 2 \mathrm{M}(\lambda=2 \pi / \mathrm{k})$ both for pinching and helical modes, above which limit a shock is expected to form. Such a shock dissipates the instability energy both to thermal and suprathermal particles; acceleration of relativistic electrons prevails for densities of the order of the typical estimates in radio jets.

The occurrence of different saturation mechanisms allows correlating observed morphology with beam parameters. Namely: a) low-luminosity beams (also "invisible" beams) are expected to be characterized by highly supersonic flow speeds; b) high- brighteness and well- defined beams are formed in supersonic flows in the presence of relatively high (long itudinal) magnetic fields; c) bright and high structured beams correspond to low Mach number flows modulated by shocks; d) subsonic flows are disrupted by instability close to the parent galactic nucleus.

We finally remark that the well- known jets in M87 and Cen A are not necess a rily to be included in this scheme, as they appear to be not in pressure equilibrium with the external medium. Most likely their morphological structures must be ascribed to instabilities in magnetically confined beams (see Ferrari and Trussoni 1981, Coppi and Ferrari 1981 for a discussion of kink resistive instabilities).

\section{References}

Benford,G.,Ferrari,A.,and Trussoni,E.: 1980, Astrophys.J.241,pp.98- 110

Ferrari,A.,Trussoni,E., and Zaninetti,L.: 1978, Astron.Astrophys.64, pp.43- 52

Ferrari,A.,Trussoni,E., and Zaninetti,L.: 1979, Astron. Astrophys. 79, pp.190- 196

Ferrari,A.,Trussoni,E., and Zaninetti,L.: 1981,Mont.Not.Royal astr.Soc., 196, in press

Ferrari,A., Trussoni,E: 1981,Proc. of the $15^{\text {th }}$ ESLAB Symp. on X-Ray Astr.,Amsterdam.

Ferrari,A.,Massaglia,S.,Trussoni,E.: 1981,Mont.Not.Royal astr.Soc,in press

Hardee,P.H.: 1979,Astrophys.J.234,pp.47- 55

Lacombe.C.: 1977,Astron.Astrophys. 54,pp. 1 - 16 